\title{
EXIGÊNCIAS PSÍQUICAS E COGNITIVAS DA EQUIPE DE ENFERMAGEM DE UM CENTRO CIRÚRGICO
}

\section{PSYCHIC AND COGNITIVE REQUIREMENTS OF AN OPERATION ROOM NURSING TEAM}

${ }^{1}$ Departamento de Prevenção e Reabilitação em Fisioterapia. Universidade Federal do Paraná,.

Autor Correspondente:

Arlete Ana Motter

E-mail: arlete.motter@uol.com.br
O artigo é fruto da iniciação científica de Flavia Cristina Miranda, com orientação da Profa. Arlete Ana Motter, que participou do projeto de pesquisa "Avaliação da Saúde dos Servidores da UFPR", no Edital PIBIC 2017/2018 da Universidade Federal do Paraná, com concessão de bolsa de iniciação científica a estudante.

\section{RESUMO}

Objetivo: analisar as exigências psíquicas e cognitivas da equipe de enfermagem de um Centro Cirúrgico (CC) e propor recomendações de melhoria nas condições de trabalho. Metodologia: Trata-se de um estudo exploratório, transversal e descritivo, com análise qualitativa dos dados. Utilizaram-se observações gerais e sistemáticas, entrevistas semiestruturadas e registros de verbalizações. A população do estudo constituiu-se por enfermeiros, técnicos e auxiliares de enfermagem que trabalharam no CC de um hospital público, localizado em Curitiba/Paraná - Brasil. Resultados: Os principais constrangimentos do trabalho da equipe de enfermagem no CC estudado relacionam-se a acúmulo de funções, falta de reconhecimento profissional, comunicação não efetiva, falta de equipamentos e insumos, sendo este o principal foco de desgaste. Conclusão: As exigências psíquicas e cognitivas dos profissionais estudados geram sobrecargas que podem afetar a saúde dos servidores, no entanto, parte das dificuldades é amenizada devido à satisfação profissional.

Palavras-chave: Enfermagem; Saúde do trabalhador; Centros cirúrgicos.

\begin{abstract}
Objective: To analyze the psychic and cognitive demands of the nursing staff of an Operation Room (OR) and propose recommendations for the improvement of working conditions. Methods: This is an exploratory, transversal, and descriptive study, with qualitative data analysis. General and systematic observations, semi-structured interviews, and verbalization records were used. The study population consisted of nurses, nursing technicians, and assistants who worked in a public hospital OR, located in Curitiba / Paraná-Brazil. Results: The main constraints from the OR nursing team studied are related to the accumulation of tasks, lack of professional recognition, uneffective communication, lack of equipment and supplies, and this is the main focus of the emotional distress. Conclusion: The psychic and cognitive demands of the professionals studied result in overloads that can affect the health of the servers, however, some of the difficulties are assuaged due to the professional satisfaction.
\end{abstract}

Keywords: Nursing; Occupational health; Surgicenters. 


\section{INTRODUÇÃO}

O Centro Cirúrgico (CC) é um setor hospitalar, com finalidade de executar procedimentos anestésicos, cirúrgicos, diag $\neg$ nósticos e terapêuticos, possuindo caráter eletivo e emergencial. constitui-se em um ambiente de intervenções invasivas e de insumos de elevada exatidão, e ainda conta com uma equipe multiprofissional; desse modo, deve haver profissionais capacitados para atender às necessidades do usuário/paciente/cliente antes, durante e após a cirurgiai ${ }^{1,2}$.

O CC apresenta-se por meio de uma área física fechada, com diversos regulamentos e rotinas, sendo assim um dos setores mais complexos do ambiente hospitalar por abranger uma elaborada logística para seu funcionamento ${ }^{3}$. Perante as suas características, faz com que usuário/paciente/ cliente estejam submetidos a diversos ris$\cos$, devido às práticas complexas e interdisciplinares, as quais se interlaçam com circunstâncias estressantes, exigindo das equipes que elas lidem com aspectos técnicos, estabilidade emocional, responsabilidade, relacionamento, insumos e a necessidade de interação entre usuário/paciente/ cliente, trabalhador e o fornecedor ${ }^{4,5}$.

No Brasil, há uma predominância de profissionais de enfermagem em hospitais, enfrentando situações diárias complexas decorrentes da relação saúde-doença ${ }^{6,7}$. $O$ profissional de enfermagem está inserido em um ambiente que demanda de atenção, atividades técnicas de elevada dificuldade, organização, administração e responsabilidade ${ }^{8}$.

Devido à elevada demanda no ambiente hospitalar, o enfermeiro está suscetível ao desgaste emocional, físico e psicológico, que é apreciado nas diferentes circunstâncias em seu trabalho'. Esses profissionais estão inseridos em um contexto peculiar, em que as condições de trabalho oferecidas são frequentemente difíceis, há excesso de trabalho, falta de recursos humanos e materiais, falta de autonomia e autoridade na tomada de decisões, relações interpessoais complicadas e necessidade de tomar decisões em tempo limitado ${ }^{10-13}$.

Esses atributos fazem com que o profissional de enfermagem tenha uma carga de trabalho muito desgastante, ritmo excessivo e jornadas extensas com insuficientes pausas para descanso, o que os torna vulneráveis ao desenvolvimento de problemas de saúde mental, pois estão submetidos a um ambiente que contribui para o desenvolvimento do estresse; portanto, a qualidade do trabalho pode refletir de forma negativa nos serviços de saúde ${ }^{8,10,14}$.

Além disso, na unidade cirúrgica, o trabalho realizado gera estresse, pois os profissionais de enfermagem permanecem nas salas de cirurgia por longos períodos, acompanhando e atuando nos múltiplos procedimentos cirúrgicos. Diante disso, o trabalhador é afetado física e emocionalmente, devido ao fato de esses profissionais gastarem energia para contrabalançar seus sentimentos, pensamentos e comportamentos. Esses fatos fazem com que ocorra um prejuízo nas relações de trabalho, no desempenho, além de ocasionarem dificuldades de atenção e concentração, queda de produtividade, desordem mental, prejuízo transitório da memória, irritabilidade, fadiga, mal-estar e acidentes ${ }^{2,15,16}$.

Assim, o interesse em desenvolver este estudo foi nor $\neg$ teado pelo objetivo de analisar as exigências psíquicas e cognitivas da equipe de enfermagem de um CC e propor recomendações de melhoria nas condições de trabalho.

\section{MATERIAIS E MÉTODOS}

A pesquisa foi desenvolvida na Unidade CC de um Hospital público localizado em Curitiba/Paraná - Brasil, sendo um hospital universitário com aulas práticas supervisionadas e estágio curricular. Trata-se de um estudo exploratório, transversal e descritivo, com análise qualitativa dos dados. 
A pesquisa desenvolveu-se entre agosto de 2017 e julho de 2018. O projeto foi apreciado pelo Comitê de Ética em Pesquisa (CEP) do Setor de Ciências da Saúde da UFPR, obtendo parecer favorável em 12 de julho de 2017, sob o número: 2168920.

A população do estudo foi composta por enfermeiros, técnicos e auxiliares de enfermagem que trabalhavam no $\mathrm{CC}$ nos turnos da manhã, tarde e noite, de ambos os sexos, possuindo como vínculos de trabalho Regime Jurídico Único (RJU), Fundação e CLT, além de apresentarem, no mínimo, 18 anos, trabalhar na Unidade CC há, no mínimo, 6 meses e ter assinado o Termo de Consentimento Livre e Esclarecido (TCLE). Do total dos profissionais que trabalham no $\mathrm{CC}$, foram excluídos aqueles que não se sentiram confortáveis em responder à entrevista, como também os que não preenchiam os critérios de inclusão.

Para obter a autorização para o início da pesquisa no $\mathrm{CC}$, foi realizada uma reunião com as enfermeiras-chefes do $\mathrm{CC}$, tendo como propósito explanar sobre como funcionariam as etapas do estudo, as quais compreenderiam em conhecer o trabalho realizado no CC para, assim, avaliar e, posteriormente, realizar as recomendações ergonômicas no ambiente de trabalho.

Na primeira etapa do estudo, foram realizadas observações gerais e conversas informais para conhecer o funcionamento e a rotina do $\mathrm{CC}$ do hospital. O contato com os participantes ocorreu no próprio turno de trabalho, individualmente ou em pequenos grupos. Essas observações foram motivadas pela Análise Ergonômica do Trabalho (AET), consistindo na análise da demanda, a qual tem por objetivo verificar o problema e sua dimensão a partir do trabalho de fato realizado. Além das observações comportamentais, é levada em conta a verbalização sobre o próprio trabalho dos profissionais. Diante disso, foi possível construir e elaborar perguntas para serem realizadas com a equipe de enfermagem.

A segunda etapa do estudo compreendeu executar entrevistas semiestruturadas; a escolha dos entrevistados foi de forma aleatória conforme a disponibilidade dos profissionais e das salas de cirurgias. $\mathrm{O}$ contato com os participantes nessa etapa também ocorreu no próprio turno de trabalho, individualmente. Os profissionais que aceitaram participar do estudo assinaram o TCLE. Os respondentes das entrevistas semiestruturadas foram identificados como E1, E2, E3, e assim sucessivamente, até E29.

Nessa mesma etapa, ocorreram registros em planilhas, sobre as ações da equipe de enfermagem, dentro da sala de cirurgia a cada 10 minutos, compreendendo, no máximo, 2 horas de observações. As ações observadas eram com relação à comunicação entre médicos e enfermeiro, técnico e auxiliar de enfermagem, entrega/abertura de materiais, organização de mesas, auxílio aos médicos, regulagem/transporte de equipamentos, preenchimento de diário, postura: sentando ou saindo da sala de cirurgia.

No total da pesquisa, foram realizadas 29 visitas a Unidade CC, resultando em 53 horas de observações, sendo que, nesse período, foram realizadas 29 entrevistas semiestruturadas e 10 registros em planilhas sobre as ações da equipe de enfermagem. Foram observadas todas as disciplinas na Unidade do CC.

\section{RESULTADO E DISCUSSÃO}

Foram realizadas, na Unidade CC, 29 entrevistas semiestruturadas, com os profissionais da equipe de enfermagem, sendo a população do estudo constituído por 5 enfermeiros, 19 técnicos de enfermagem e 5 auxiliares de enfermagem. Para melhor compreensão do fenômeno pesquisado, a totalidade da amostra foi composta por profissionais do sexo feminino $(69 \%)$ e masculino (31\%), apresentando idade entre 27 e 59 anos.

A respeito do local da pesquisa, observou-se uma complexa articulação entre diversas empresas e entre diversas categorias profissionais. Diante disso, esse local caracteriza-se por conter 14 salas de cirurgias, e a disposição das cirurgias em determinadas salas ocorre devido a materiais específicos de certa especialidade (quadro 1). A configuração do CC ocorre da seguinte maneira:
Miranda e Motter 


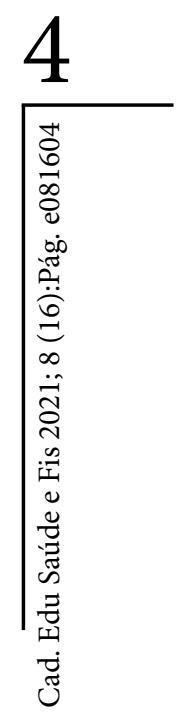

Quadro 1. Distribuição de Disciplinas nas Salas Cirúrgicas

\begin{tabular}{|l|l|}
\hline Salas & \multicolumn{1}{|c|}{ Disciplinas } \\
\hline Sala 1 & Cirurgia geral, Coloproctostomia e atualmente pediatria \\
\hline Sala 2 & Cirurgia oftalmológica \\
\hline Sala 3 & Sala de equipamentos \\
\hline Sala 4 & Ortopedia \\
\hline Sala 5 & Cirurgia otorrinolaringológica, Cirurgia Torácica e Cardíaca. \\
\hline Sala 6 & Sem especialidade definida \\
\hline Sala 7 & Cirurgia plástica \\
\hline Sala 8 & Equipamentos \\
\hline Sala 9 & Cirurgia cardíaca e torácica \\
\hline Sala 10 & Urologia \\
\hline Sala 11 & Neurocirurgia \\
\hline Sala 12 & Cirurgia geral \\
\hline Sala 13 & Cirurgia pediátrica - interditada \\
\hline Sala 14 & Cirurgia pediátrica \\
\hline
\end{tabular}

Ainda, há algumas salas específicas para o armazenamento de materiais e equipamentos; sala arsenal (sala contendo materiais a serem utilizados em cirurgias de emergência ou intercorrência cirúrgica); sala de órteses e próteses; farmácia; sala exclusiva para torres de vídeo; sala dos anestesistas; sala de conforto para os profissionais, sala de reuniões, expurgo; sala de peças anatopatológicas e de processamento de materiais que não precisam ser necessariamente esterilizados - e, ainda, o CC é constituído pela sala de recuperação pós-anestésico (Repai).

\section{Os diferentes papéis do enfermeiro na Unidade CC}

Para que as atividades do CC sejam concretizadas, é imprescindível que se tenha um trabalho integrado, com profissionais qualificados e preparados, devido às exigências impostas pelo ambiente ${ }^{17}$. Diante desse fato, é necessário que haja uma equipe de enfermagem, a qual é constituída por gerenciadores, circulantes, profissionais do transporte (maqueiros) e assistência na Repai.
As atividades gerenciais possuem o desafio de organizar as diferentes interfaces do trabalho, tendo o intuito de assegurar a qualidade da assistência de enfermagem e o bom funcionamento da unidade por meio da verificação das salas operatórias disponíveis e anestesistas, entrega de materiais no prazo previsto e disponibilidade dos materiais, verificando o agendamento de cirurgias e fazendo com que as cirurgias ocorram em horários predeterminados, capacitação para a equipe de enfermagem, administração dos recursos humanos, dimensionamento da equipe e planejamento - sendo essas funções semelhantes ao apontado no estudo de Stumm et al. e Giordani et al. ${ }^{3,18}$.

Segundo a Associação Brasileira de Enfermeiros de Centro Cirúrgico, Recuperação anestésica e Centro de Material e Esterilização (Sobecc), o enfermeiro gerenciador de $\mathrm{CC}$ atua como um gerente burocrático, organizacional e assistencial. Nesse sentido, o bom funcionamento do $\mathrm{CC}$ encontrar-se diretamente condicionado a um eficiente gerenciamento desempenhado pelo enfermeiro ${ }^{19}$.

As atividades desempenhadas pelo circulante no hospital observado possuem a seguinte descrição: observar o mapa de cirurgias do dia; buscar na farmácia os materiais a serem utilizados no procedimento previsto; verificar o carrinho da 
Central de Materiais (CM), observando se os instrumentais vieram do $\mathrm{CM}$ e se os campos estão íntegros e secos, se têm etiquetas e se foram esterilizados em autoclave.

Após a verificação dos materiais, a sala na qual será realizada a cirurgia deve ser organizada, ou seja, ligar o ar-condicionado, passar compressa com álcool em todos os móveis da sala, checar o aspirador, checar as lâmpadas (caso alguma lâmpada esteja queimada, a circulante deve providenciar um spot móvel). Em seguida, a circulante verifica para o anestesiologista se tem traqueia, cânulas, bandeja, balões, conexões etc., abre o LAP, confere o checklist pré-operatório do paciente e, após esse processo, avisa ao maqueiro para buscar o paciente.

Depois que a cirurgia teve início, o circulante fica à disposição de todos que entram em campo. Durante a cirurgia, permanece sentado, mas atende a equipe no que necessitar - caso falte algum insumo, precisa sair da sala e buscar, além de preencher o checklist transoperatório. Após o término do procedimento, o circulante vai até a Repai, seleciona uma cama e traz até a sala de cirurgia, e ajuda a fazer a transferência do paciente (da mesa cirúrgica para a cama). Em seguida, pega no expurgo a caixa de armazenamento dos materiais utilizados no procedimento e organiza a sala.

O maqueiro possui a função de transportar o paciente de uma unidade para outra do hospital. Em observações informais, pode-se notar que as enfermeiras que estão na sala da Repai precisam preencher um checklist sobre o pós-operatório imediato, dando uma nota para o paciente de zero a 2, para a atividade motora, respiratória, circulatória, consciência, $\mathrm{SpO} 2$, cor, dor e náuseas/vômitos do paciente.

\section{Sobrecarga de trabalho da equipe de enfermagem}

A ergonomia cognitiva avalia aspectos emocionais e mentais, enquanto a ergonomia organizacional abrange tópicos de políticas, processos e gestão do sistema produtivo. Uma das etapas que compreendem a ergonomia é com relação à análise da tarefa, sendo um conjunto de objetivos prescritos que os trabalhadores devem cumprir. A AET analisa a discrepância entre a tarefa que é prescrita (descrição de cargos) e os requisitos físicos para execução da tarefa ${ }^{21}$.

O CC está inserido em um cenário com diversas demandas de trabalhos específicas. Advém que a equipe de enfermagem se encontra submetida a um acúmulo de funções, sendo um problema apontado pelos entrevistados, ou seja, enfermeiros/ técnicos/auxiliares são contratados para desempenhar uma determinada função e completam outras atividades, acontecendo uma diferença entre o trabalho prescrito e o real. Sobre tais situações, convém pontuar:

"Antigamente o circulante só circulava, agora assumem serviços da central de materiais e do transporte de paciente" (E10).

"Realizo o gerenciamento daqui, mas muitas vezes faço a função do enfermeiro, técnico de enfermagem, de maqueiro e fico dentro de sala (circulante) [...] quando há muitas cirurgias acontecendo" (E17).

"[...] há um acúmulo de função, precisa realizar três a quatro coisas que não seria da sua função" (E1).

\section{As comunicações entre os profissionais do $C C$}

A comunicação contempla um processo no qual está envolvida em transferências e compartilhamentos verbais e não verbais, caracterizando-se como um componente fundamental no cuidado. Diante disso, é relevante que nele ocorra o compartilhamento para obter um relacionamento interpessoal $^{22}$.

Os enfermeiros que exercem suas atividades no CC não trabalham isoladamente; e o fato de serem profissionais heterogêneos pode ser um dos fatores geradores de conflitos, divergências, insatisfações, levando à desintegração e a dificuldades relacionais entre a equipe ${ }^{3,23}$.

Os resultados das entrevistas com os profissionais mostraram que muitos evitam 
abordar o assunto sobre a comunicação e o relacionamento entre os profissionais no ambiente de trabalho. Diante desse fato, apenas 58\% dos entrevistados responderam a essa temática, sendo que, desses, $42 \%$ consideraram sendo boa a comunicação e o relacionamento entre os funcionários da enfermagem e demais profissionais, e $59 \%$ desses profissionais ponderaram que, em determinadas situações, não é efetiva, acarretando problemas para organização da unidade do CC. As frases abaixo ilustram tais situações: (E5).

"Boa, a gente consegue se entender"

“É boa. Mas às vezes temos desentendimentos. [...] Não gosto que me apressem, e fiquem falando o que eu tenho que fazer, porque eu sei. Ficam falando abre o cautério, liga a máquina" (E10).

"Pode ser considerada como boa, claro que tem uns que não são muitos abertos, não deixam a gente muito confortáveis para a conversa, mas eu considero médio, razoável" (E21).

A relação interpessoal é um aspecto que interfere psicologicamente no profissional. Desse modo, desentendimentos entre os profissionais interferem na continuidade, na qualidade e no êxito do trabalho, comprometendo diretamente na execução do funcionamento da unidade e no cuidado ao paciente, além de gerar danos à saúde desses profissionais ${ }^{3,24}$. Um relacionamento adequado entre os profissionais operantes no CC é primordial para o bom andamento das cirurgias e, também, estimula um ambiente de cuidado harmônico ${ }^{3}$.

A partir dos registros em planilhas sobre as ações da equipe de enfermagem dentro da sala de cirurgia, verifica-se que a comunicação tem sua importância entre os profissionais. A média do tempo de observação das cirurgias foi de 52,5 minutos, sendo que a comunicação dentro desse ambiente ocorreu em média, 20,4 vezes. Dessa forma, pode-se dizer que ocorre um diálogo entre os membros da equipe e o enfermeiro, em média, a cada 2,6 minutos.

Outro aspecto que podemos observar com o registro em planilhas consiste na relação da distribuição de tarefas por meio do número de repetições. Durante a preparação do paciente para a cirurgia, ocorre uma intensificação da abertura e entrega de materiais, organização de mesas e comunicações. Após o início do procedimento cirúrgico, há uma redução de repetição dessas tarefas e intensificação do preenchimento do diário cirúrgico, assim como aumento do tempo onde a circulante permanece sentada. Quanto aos auxílios aos médicos e saídas às salas cirúrgicas, essas permanecem equilibradas após início da cirurgia. A comunicação adequada é fundamental para garantir a segurança nos procedimentos realizados no $\mathrm{CC}$, bem como a saúde do trabalhador.

\section{Condições de trabalho}

A análise dos resultados apontou problemas com relação à falta de insumos e de equipamentos no $\mathrm{CC}$, além de ocorrer desperdícios desses materiais, pois muitos funcionários abrem materiais a mais durante a cirurgia que não são usados; com isso, a sobra desses insumos não pode ser guardada, e eles são descartados. Outros aspectos relacionados com esse assunto são com relação a materiais sucateados, falta de medicamentos, maca impropria sem regulagem, falta de UTI, transferidor de paciente (Skate) e guindaste, falta cadeiras adequadas dentro das salas de cirurgia, barulho. Diante dos aspectos apontados pelos entrevistados, podemos correlacioná-los com as condições inadequadas e precarização do trabalho realizados pela equipe de enfermagem.

As condições/precarizações de trabalho são caracterizadas como uma situação atípica e desfavorável à saúde do trabalhador, ou seja, está associada com informações e circunstâncias que se inter-relacionam, em um ambiente inadequado à saúde dos profissionais de saúde ${ }^{25}$.

Outro aspecto apontado nas entrevistas semiestruturadas está relacionado com o problema de recursos humanos insuficientes, pressão, problema com o vínculo de trabalho, aspectos esses que são resultados de fatores organizações, capazes de gerar sofrimento aos trabalhadores.

Com relação aos vínculos de trabalho 
do CC, os entrevistados do vínculo RJU (concursados) apontaram que os vínculos que são regidos por CLT são considerados pelos funcionários como mais tranquilos, o que não seria o caso deles. No entanto, os trabalhadores regidos por CLT (terceirizados) assinalam nas entrevistas que possuem uma "rédea mais curta", além de apresentarem piores questões administrativas. Além disso, existem diferenças salariais: por exemplo, a empresa privada que presta serviços ao hospital, contratos CLT, paga melhor $(40 \%)$ do que a RJU $(20 \%)$ pelas condições de trabalho considerando as substâncias químicas, às quais os profissionais estão expostos naquele setor.

De acordo com a Sociedade Brasileira de Enfermeiros de Centro Cirúrgico, o desenvolvimento da tecnologia em hospitais, com relação aos equipamentos e matérias, fez com que complementasse a qualidade do serviço prestado no CC, viabilizando ao enfermeiro, dessa forma, um ambiente favorável para o desenvolvimento do cuidado, diminuindo assim os riscos e as complicações ${ }^{20}$.

Por outro lado, a falta de material leva a uma desorganização do $\mathrm{CC}$ e a um desgaste do trabalhador, pois, quando não há um insumo, as cirurgias podem ser canceladas, uma vez que, sem materiais de segurança, não tem como aprovar o início do procedimento cirúrgico. No entanto, geralmente, quando falta um material, ocorre um improviso para a situação. Esses problemas geram impactos e afetam diretamente o trabalho. Diante do apresentado, percebe-se que deve haver um maior controle perante os materiais, podendo assim diminuir os custos e o estresse.

"[...] não há conflitos apenas entre enfermeiros e médicos e sim da equipe de forma geral, sendo a causa mais comum os materiais" (E14).

Esse comentário ilustra como a falta de insumos em um CC pode ser um dos focos de desconforto e estresse no ambiente de trabalho. Entre os profissionais da equipe de enfermagem, ao serem questionados sobre qual seria o principal foco de estresse, $35,29 \%$ dos entrevistados apontaram como sendo a falta de materiais, conforme o registro das falas seguintes:

"A falta de materiais, sempre gera estresse para o circulante, pois temos que providenciar, ainda mais se o médico já tiver iniciado o procedimento, mas claro que tem situações que fogem do nosso alcance" (E9).

"A falta de material. Antes eu trabalhava nas salas com a ortopedia e pedi pra sair justamente por essa questão. Era muito estressante e desgastante a falta de material para a cirurgia e a gente vê que para resolver a situação era preciso só um pouquinho de boa vontade e as pessoas não tinham isso" (E14).

"A falta de materiais. A central de materiais (CM) é o coração do hospital e os gestores não valorizam isso. Colocam os profissionais mais velhos para trabalhar lá, como se eles não fossem mais úteis aqui no CC ou na enfermaria, ai colocam na CM. Mas na verdade a CM precisa de profissionais jovens e ágeis trabalhando porque senão acontece o que está acontecendo agora. Atrasa o material, interrompe cirurgia e aí atrasa o próximo procedimento" (E18).

Segundo a tabela de registro, o circulante sai, em média, 6,9 vezes da sala para buscar determinado material, diante de uma cirurgia que perdura em média 52,5 minutos, ou seja, a cada 7,6 minutos de cirurgia o circulante sai da sala para buscar um insumo. 


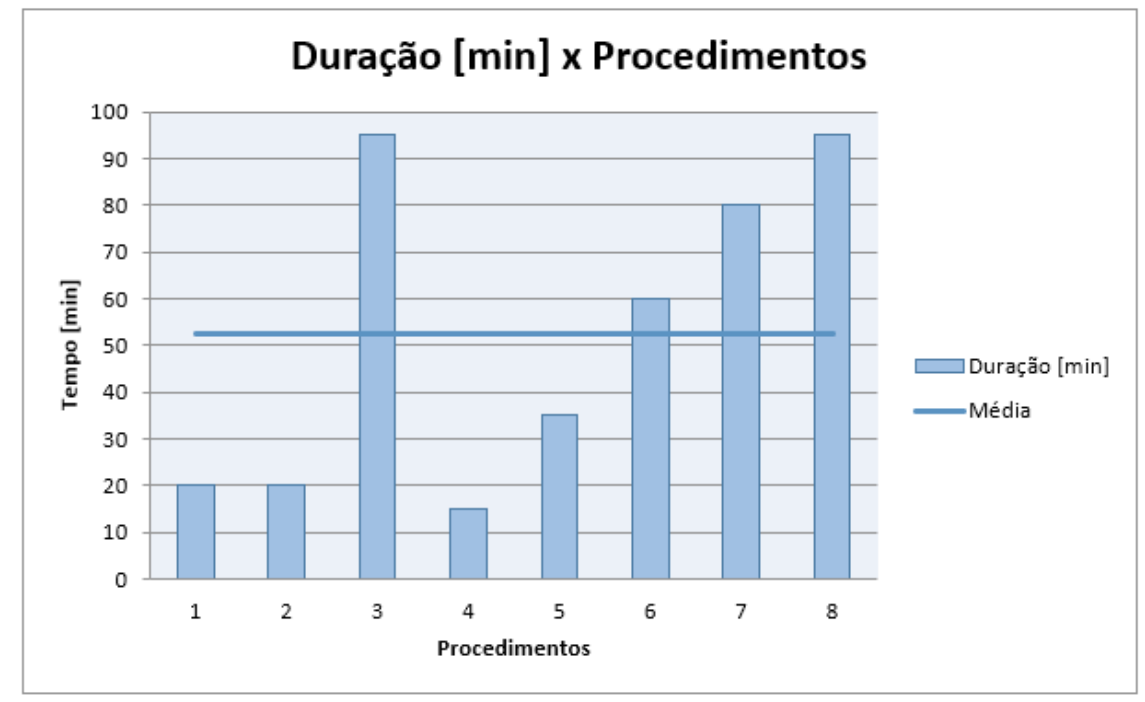

Fonte: elaboração própria.

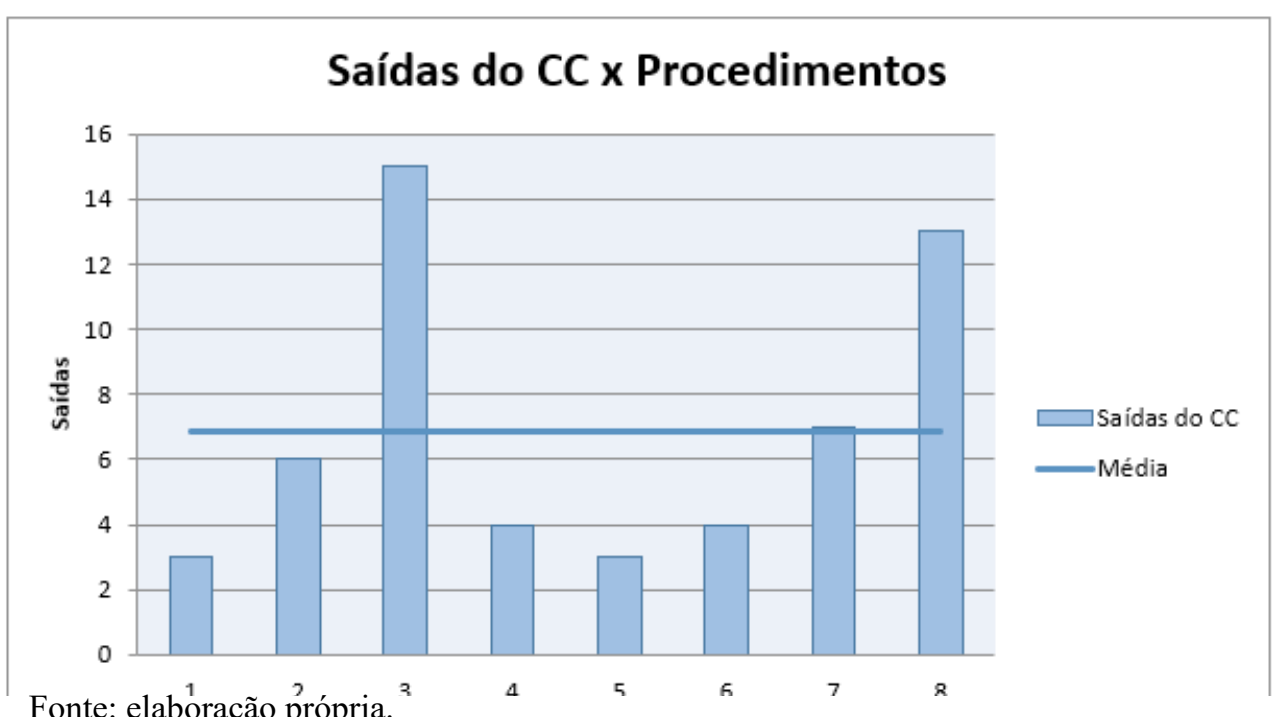

Fonte: elaboração própria. ${ }^{3}$ 
No entanto, além da falta de materiais, foram citados como fatores estressores: os residentes, os relacionamentos interpessoais, a discussão entre membros da equipe, a desorganização, o acúmulo de função, as macas e os pontos de trabalhos que são longe.

"Os residentes são muito afobados e querem fazer cirurgia sem material, fazer com o que tem e não pensam no paciente. O que é estressando é a pressão que fazem em cima da gente pra que a cirurgia aconteça. Parece que eles querem produtividade sabe? Mas produtividade sem segurança não dá... Já tive paciente que voltou pro CC por infecção, por exemplo" (E26).

Identificou-se também que esse estresse ocasionando no ambiente de trabalho acarreta, ao final do expediente, um maior cansaço mental na equipe de enfermagem, pois os funcionários estão constantemente raciocinando no que pode ocorrer na cirurgia e o que precisa utilizar na próxima cirurgia. Portanto, há um aumento da pressão; e, assim, o processo de trabalho leva a um cansaço mental dos enfermeiros/ técnicos/auxiliares.

\section{Reconhecimento profissional}

Um ponto negativo encontrado nas entrevistas está relacionado com o reconhecimento do serviço dos enfermeiros/ técnicos/auxiliares na unidade do $\mathrm{CC}$, $68,75 \%$ dos entrevistados asseguram não ter recebido qualquer reconhecimento do seu serviço na unidade e $31,25 \%$ garantem que há reconhecimento do seu trabalho por parte dos colegas e pacientes. Percebe-se, nesse sentido, que o trabalho desses profissionais acaba sendo visto como um trabalho complementar, e que possui pouca ou nenhuma autonomia, o que conjetura na invisibilidade do profissional, conforme as falas seguintes:

"Não tem e vai estressando, magoando. Trabalho há anos e nunca tive nada de reconhecimento" (E1).

"Mais ou menos [...] muitos dizem que a enfermagem é um mal necessário" (E10).
"Nenhum. Vez ou outra há médicos que reconhece, mas a maioria não. Nem aqui e nem em outros locais. Somos tratados nem como $3^{a}$ categoria e sim $5^{a}$ categoria" (E19)

Por meio das falas mencionadas acima, tornar-se claro que é imprescindível incentivar esses profissionais, assim como a sua autonomia, além de contribuir para um maior reconhecimento, valorização. Dessa forma, contribuirá para uma maior visibilidade da profissão.

\section{Satisfação profissional}

A satisfação profissional é um fenômeno subjetivo, que consiste em uma atitude, uma emoção ou um sentimento agradável ou positivo, que pode ser suficiente para definir o ambiente de trabalho ${ }^{26,27}$. Devido à satisfação profissional ser subjetiva, a conjuntura de satisfação ou insatisfação pode modificar as condições gerais de trabalho ${ }^{26}$.

Mesmo com as exigências/dificuldades apresentadas pelos entrevistados no $\mathrm{CC}$, quando questionados sobre a satisfação em relação à profissão, eles demonstraram estar felizes com a profissão que escolheram e que gostavam da unidade.

"Não sei se outra profissão iria me satisfazer" (E5).

"Gosto do que eu faço bastante e gosto do setor também, pois aqui no centro cirúrgico você não chega a se envolver, o paciente chega faz a cirurgia e vai embora e tudo bem" (E24).

Enfermeiros que executam suas atividades em um meio considerado favorável à prática profissional proporcionam experiências positivas de trabalho. Os aspectos que favorecem a satisfação profissional para os profissionais de enfermagem recaem sobre autonomia, produtividade, quantidade do trabalho, êxito, salário, relação interpessoal, satisfação dos pacientes, valorização e reconhecimento, possibilidade de amenizar o sofrimento e melhorar a saúde do paciente, além de aspectos 
envolvendo suporte organizacional, boas não se dá de forma adequada, sendo um condições de infraestrutura, materiais e aspecto negativo que contribui para um recursos humanos ${ }^{28-30}$. ambiente desgastante e exaustivo. Nesse caso, a própria gerência da unidade e a

As particularidades geradoras de desgaste e insatisfação profissional ao enfermeiro em um hospital compreendem falta e/ou insuficiência de equipamentos e mategestão hospitalar necessitam pensar em estratégias para melhorar a integração entre membros das equipes e profissioriais, falta de profissionais, sofrimento e de trabalho. Quanto à falta de reconhemorte do paciente, sobrecarga de trabalho cimento profissional, este um aspecto e condições precárias, falta de reconheci- negativo no trabalho da enfermagem que mento profissional e valorização das ati- precisa ser modificado, para permitir que vidades exercidas. Esses elementos levam esses profissionais saiam da invisibilidade o profissional à exaustão física e mental, e que sejam valorizados e prestigiados em além de baixa autoestima ${ }^{28-30}$. um trabalho tão importante para usuário/ paciente/cliente, para a instituição e para a sociedade.

\section{CONCLUSÃO}

\section{REFERÊNCIAS}

A equipe de enfermagem frequente-

1. Carvalho PA, Göttems LBD, Pires mente enfrenta no CC exigências relacio- MRGM, Oliveira MLC. Safety culture in nadas com o ambiente hospitalar de alta complexidade. Diante disso, ao final da jornada de trabalho, os profissionais estão suscetíveis a um desgaste mental, como apontado nos resultados desta pesquisa, podendo, assim, resultar em sofrimento no trabalho e comprometer a saúde dos servidores.

A pesquisa evidencia que os participantes enfrentam várias dificuldades, incluindo conflitos interpessoais, falta de materiais e insumos, falta de reconhecimento, entre outros, que acarretam desestruturação na dinâmica do funcionamento da unidade, comprometendo a qualidade do atendimento ao paciente. A deficiência de recursos humanos e de insumos leva enfermeiro nas atividades gerenciais. ao acúmulo de tarefas, à precarização do Rev Gaúcha Enferm [Internet]. 2016 trabalho e à desorganização do CC. Reco- [acessado 2017 out 26];37(4):e56945. menda-se controle de materiais por meio Disponível em http://www.scielo.br/ de planilha de fluxo com a finalidade de scielo.php?script=sci_arttext\&pid=S1983evitar desperdícios e de melhorar o geren- $14472016000400415 \& \operatorname{lng}=\mathrm{en}$.

ciamento. Também sugere-se investir na compra de insumos. Outrossim, a sobrecarga de trabalho poderia ser amenizada com a contratação de novos funcionários para a unidade e com a introdução de pausas para descanso durante a jornada de trabalho.

5. Fernandes HMLG, Peniche ACG. Percepção da equipe de enfermagem do Centro Cirúrgico acerca da Acreditação Hospitalar em um Hospital Universitário. Rev Esc. Enferm USP. 2015;49(spe):22-28.

Pode-se identificar, também, que a 6. Stacciarini JMR, Tróccoli BT. O comunicação da equipe de enfermagem estresse na atividade ocupacional do 
enfermeiro. Rev Latino-Am Enfermagem. 2001;9(2):17-25

7. Albendín L, Gómez JL, Lafuente GAC, Cañadas GR, Luis CS, Aguayo R. Prevalencia bayesiana y niveles de burnout en enfermería de urgencias. Una revisión sistemática. Rev Latino-Am Psicología. 2016;48(2):137-145.

8. Rocha MCP, Martino MMF. O estresse e qualidade de sono do enfermeiro nos diferentes turnos hospitalares. Rev Esc Enferm USP. 2010;44(2):280-286.

9. Fonseca JRF, Lopes Neto D. Levels of occupational stress and stressful activities for nurses working in emergency. Rev Rede. 2014;15(5):732-742.

10. Quintas S, Queirós C, Marques A, Orvalho V. Os enfermeiros e a sua saúde no trabalho: a relação entre depressão e burnout. International Journal on Working Conditions. 2017;(13):1-20.

11. Garrosa E, Rainho C, Moreno-Jiménez B, Monteiro MJ. The relationship between job stressors, hardy personality, coping resources and burnout in a sample of nurses: A correlational study at two time points. Int $\mathrm{J}$ Nurs Stud. 2010;47(2):205-215.

12. Ferreira LI, Duarte TESS, Filho PSG, Assis EV, Feitosa ANA, Sousa MNA. Estresse no Cotidiano de Trabalho dos Enfermeiros da Urgência e Emergência. Revista Interdisciplinar em Saúde. 2016;3(1):108-128.

13. Jofre AV, Valenzuela SS. Burnout en personal de enfermería de la Unidad de Cuidados Intensivos Pediátricos. Aquichan. 2005;5(1):56-63.

14. Lorenz VR, Benatti MCC, Sabino MO. Burnout and Stress Among Nurses in a University Tertiary Hospital. Rev Latino-Am Enfermagem. 2010;18(6):1084-1091.

15. Smeltzer SC, Bare BG, Hinkle JL, Cheever KH. Brunner \& Suddarth - Tratado de enfermagem médico-cirúrgica. 11. ed. Rio de Janeiro: Guanabara Koogan; 2008
16. Lipp M, Malagris L. Mitos e Verdades sobre o Stress. São Paulo: Contexto; 1996.

17. Caregnato RCA, Lautert L. O estresse da equipe multiprofissional na Sala de Cirurgia. Rev Bras Enferm. 2005;58(5):545-550.

18. Giordani JN, Bisogno SBC, Silva LAA. Percepção dos enfermeiros frente às atividades gerenciais na assistência ao usuário. Acta Paul Enferm. 2012;25(4):511-516.

19. Hausmann M, Peduzzi M. Articulação entre as dimensões gerencial e assistencial do processo de trabalho do enfermeiro. Texto Contexto - Enferm. 2009;18(2):258-265.

20. Associação Brasileira de Enfermagem de Centro Cirúrgico Recuperação Anestésica e Centro de Material e Esterilização (SOBECC). Práticas Recomendadas SOBECC. 6. ed. São Paulo: Manole; 2013.

21. Iida I, Buarque L. Ergonomia: Projeto e Produção. 2. ed. São Paulo: Edgard Blucher; 2005.

22. Diógenes MAR, Linard AG, Teixeira CAB. Comunicação, acolhimento e educação em saúde na consulta de enfermagem em ginecologia. Revista Rene. 2010;11(4):38-46.

23. Silva MJP. Comunicação tem remédio: a comunicação nas relações interpessoais em saúde. São Paulo: Editora Loyola; 2010 .

24. Broca PV, Ferreira MA. Processo de comunicação na equipe de enfermagem fundamentado no diálogo entre Berlo e King. Esc Anna Nery. 2015;19(3):467-474.

25. Amorim LKA, Carvalho CA, Souza NVDO, Cruz ESER, Silva MGV. $\mathrm{O}$ trabalhador sem vínculo previdenciário e a vivência cirúrgica: uma contribuição da enfermagem. Cienc Cuid Saúde. 2012;11(2):319-327.

26. Melo MB, Barbosa MA, Souza PR. Satisfação no trabalho da equipe de enfermagem: revisão integrativa. Rev Latino-Am Enfermagem. 2011;19(4):1047-1055. 
27. Locke EA. The nature and causes of job satisfaction. In: Dunnette MD, editor. Handbook of industrial and organizational psychology. Chicago: Rand McNally College Publishing; 1976. p. 1297-349.

28. Gallo CMC. Desvelando fatores que afetam a satisfação e a insatisfação o trabalho de uma equipe de enfermagem [dissertação]. Rio Grande (RS): Universidade Federal do Rio Grande; 2005.

29. Kessler AI, Krug SBF. Do prazer ao sofrimento no trabalho da enfermagem: o discurso dos trabalhadores. Rev Gaúcha Enferm. 2012;33(1):49-55.

30. Somense CB, Duran ECM. Hygiene and motivation factors of nursing work in a cardiology ward. Rev Gaúcha Enferm. 2014;35(3):82-89.

Recebido: $18 / 03 / 2020$

Aprovado: 30/09/2020 